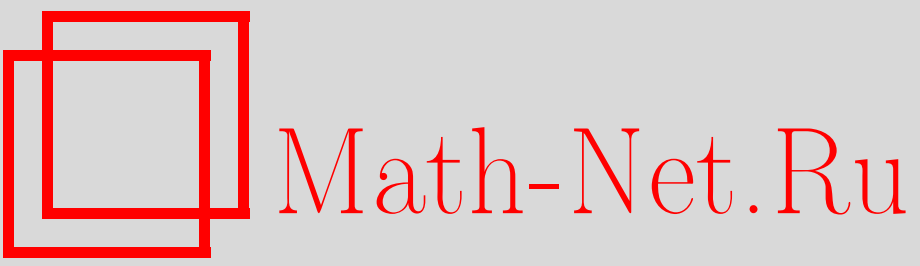

М. А. Шубарин, Условия неизоморфности пары весовых пространств непрерывных функций, Итоги науки и техн. Сер. Соврем. мат. и ее прил. Темат. обз., 2021, том 190, 144-155

DOI: https://doi.org/10.36535/0233-6723-2021-190-144-155

Использование Общероссийского математического портала Math-Net.Ru подразумевает, что вы прочитали и согласны с пользовательским соглашением

http://www. mathnet.ru/rus/agreement

Параметры загрузки :

IP: 3.80 .181 .102

26 апреля 2023 г., 03:23:41 


\title{
УСЛОВИЯ НЕИЗОМОРФНОСТИ ПАРЫ ВЕСОВЫХ ПРОСТРАНСТВ НЕПРЕРЫВНЫХ ФУНКЦИЙ
}

\author{
(c) 2021 г. $\quad$ М. А. ШУБАРИН
}

\begin{abstract}
АннотАция. В статье формулируются условия неизоморфности пары пространств Фреше. Эти условия формулируются в терминах инвариантных классов пространств $\left(D_{1}\right)$ и $\left(D_{2}\right)$. В качестве следствия доказывается неизоморфность весовых пространств непрерывных функций специального вида. Эти пространства можно рассматривать как модификацию пространств степенных рядов конечного и бесконечного типа.
\end{abstract}

Ключевъе слова: изоморфные пространства, ограниченный оператор, метод вещественной интерполяции, весовое пространство непрерывных функций.

\section{CONDITIONS FOR THE NONISOMORPHISM \\ OF A PAIR OF WEIGHTED SPACES \\ OF CONTINUOUS FUNCTIONS}

\author{
(c) 2021 M. A. SHUBARIN
}

\begin{abstract}
In this paper, we formulate conditions for a pair of Fréchet spaces to be nonisomorphic. These conditions are formulated in terms of invariant classes of the spaces $\left(D_{1}\right)$ and $\left(D_{2}\right)$. As a consequence, we prove that weighted spaces of continuous functions of a special form are nonisomorphic. These spaces can be considered as a modification of spaces of power series of finite and infinite type.

Keywords and phrases: isomorphic spaces, bounded operator, real interpolation method, weighted space of continuous functions.
\end{abstract}

AMS Subject Classification: 47B10, 46B70, 46A63

\section{1. Введение. Постановка задачи.}

1.1. В статье рассматривается проблема изоморфной классификации пространств Фреше и, в частности, весовых пространств непрерывных функций.

Пространства Фреше (т.е. полные метризуемые локально выпуклые пространства) $X$ и $Y$ называют изоморфными (и пишут $X \cong Y$ ), если существует непрерывный биективный линейный оператор $T: X \rightarrow Y$. Оператор $T$, удовлетворяющий перечисленным условиям, называют изоморфизмом. Если не существует изоморфизма $T: X \rightarrow Y$, то говорят, что пространства $X$ и $Y$ неизоморфны и пишут $X \not Y$.

Проблема изоморфной классификации состоит в нахождении условий изоморфности или неизоморфности двух пространств Фреше. 
1.2. Практикуется два подхода к решению этой проблемы. Первый из них основан на изучении линейных топологических инвариантов. По определению, линейным топологическим инвариантом (сокращённо - ЛТИ) называют произвольное отображение $\Psi$, заданное на некотором множества $\mathcal{K}$ пространств Фреше и удовлетворяющее следующему условию: если пространства Фреше $X, Y$ из $\mathcal{K}$ изоморфны, то $\Psi(X)=\Psi(Y)$. Таким образом, в терминах ЛТИ можно сформулировать необходимое условие изоморфности пространств из $\mathcal{K}$. Представляет интерес выделить в $\mathcal{K}$ подмножество $\mathcal{K}_{0}$, на котором $\Psi$ будет сильнейшим линейным топологическим инвариантом. Другими словами, из равенства $\Psi(X)=\Psi(Y)$ для пространств из $\mathcal{K}_{0}$ должна следовать изоморфность этих пространств.

Нетривиальными ЛТИ являются:

(1) аппроксимативная размерность (см. [7,12,17]);

(2) диаметральная размерность (см. [11]) и её модификации (см. [8], дальнейшие обобщения и библиография в [24]).

1.3. Другой подход к решению проблемы изоморфной классификации состоит в нахождении условий, при выполнении которых ни один линейный оператор, действующий в рассматриваемых пространствах, не будет изоморфизмом.

Условия, из которых следует неизоморфизм оператора $T$, можно сформулировать в терминах «малости» образа этого оператора.

Самое сильное условие малости образа оператора - конечномерность образа этого оператора.

Теорема 1.1. Пусть пространство Фреше $X$ бесконечномерно и произвольный линейный оператор $T: X \rightarrow Y$ имеет конечномерный образ. Тогда пространства $X$ и $Y$ не изоморфны.

Доказательство этого утверждения основано на том, что при сделанных предположениях $X$ или $Y$ конечномерно.

Более сильное условие неизоморфности формулируется в терминах компактных операторов: линейный оператор $T: X \rightarrow Y$ называют компактным, если в $X$ существует окрестность нуля $U$ такая, что её образ $T U$ будет относительно компактным подмножеством в $Y$.

Определение 1.1 (Захарюта В. П. [23]). Пространства Фреше $X, Y$ называют сильно различными (и пишут $(X, Y) \in R$ ), если любой линейный непрерывный оператор $T: X \rightarrow Y$ компактен.

Теорема 1.2. Сильно различные бесконечномерные пространства Фреше не изоморфны.

1.4. Таким образом, доказательство неизоморфности пространств $X$ и $Y$ сводится к нахождению условий сильного различия этих пространств. Один из вариантов этих условий был получен В. П. Захарютой [23] для пространств Кёте и были сформулированы в терминах инвариантных классов $\left(d_{1}\right)$ и $\left(d_{2}\right)$.

Бесконечную матрицу $A=\left(a_{p, n}\right)_{p, n=1}^{+\infty}$ называют матрицей Кёте, если выполняются следующие условия: $0<a_{p, n} \leqslant a_{p+1, n}$ для произвольных натуральных чисел $p$ и $n$. Пространством Кёте называют векторное пространство

$$
K(A):=\left\{x=\left(x_{n}\right) \in \omega: \forall p\|x\|_{p}:=\sum_{n=1}^{\infty}\left|x_{n}\right| a_{p, n}<+\infty\right\} .
$$

Набор норм $\left(\|\cdot\|_{p}\right)$ задаёт в $X$ топологию пространства Фреше.

Пусть $K(A)$ - пространство Кёте. Говорят, что пространство $K(A)$ имеет тип $\left(d_{1}\right)$ и пишут $K(A) \in\left(d_{1}\right)$, если

$$
\exists p_{0} \forall p \forall \tau \in(0,1) \exists p_{1}: \exists C>0 \forall n \in \mathbb{N} a_{p, n} \leqslant C a_{p_{0}, n}^{\tau} a_{p_{1}, n}^{1-\tau} .
$$

Говорят, что пространство $K(A)$ имеет тип $\left(d_{2}\right)$ и пишут $K(A) \in\left(d_{2}\right)$, если

$$
\forall q_{0} \forall \tau \in(0,1) \exists q \forall q_{1}: \exists C>0 \forall n \in \mathbb{N} a_{q, n} \geqslant C^{-1} a_{q_{0}, n}^{1-\tau} a_{q_{1}, n}^{\tau} .
$$


Классы пространств $\left(d_{j}\right)$ в терминах поперечников Колмогорова были определены М. М. Драгилевым [2]. Приведённые выше условия (без дополнительных ограничений на пространства) содержатся в работе В. П. Захарюты [23].

Теорема 1.3 (Захарюта В. П. [3, теорема 2], [23]). Пусть выполняются следующие условия:

(1) $X \cong K(A), Y \cong K(B)$;

(2) $X$-монтелевское пространство;

(3) $K(A) \in\left(d_{2}\right), K(B) \in\left(d_{1}\right)$.

Тогда пространства $X$ и $Y$ сильно различнь. В частности, эти пространства не изоморфны.

Пример 1.1. Пусть $0<r \leqslant+\infty$. Через $A_{r}$ обозначают пространство функций, аналитических в круге $|z|<r$. Топология пространства Фреше в этом пространстве определяется как топология равномерной сходимости на компактных подмножествах в круге $|z|<r$. Пространства $A_{r}$ не являются пространствами Кёте, но в них есть абсолютный базис и поэтому они изоморфны подходящим пространствам Кёте. Известно, что

(1) $A_{1}$ изоморфно пространству Кёте из $\left(d_{2}\right)$,

(2) $A_{\infty}$ изоморфно пространству Кёте из $\left(d_{1}\right)$,

(3) $A_{\infty}$ - монтелевское пространство.

Неизоморфность пространств $A_{1}$ и $A_{\infty}$ есть следствие перечисленных свойств и теоремы 1.3.

Рассматриваемые в примере пространства интересны тем, что их можно рассматривать в качестве контрпримера к следующей гипотезе: если пространства $X, Y$ таковы, что $(X, Y) \in R$, следует ли отсюда, что $(Y, X) \in R$ ? Для доказательства того, что $(Y, X) \notin R$ достаточно заметить, что $A_{\infty}$ изоморфно подпространству в $A_{1}$ (см. $[18$, лемма 2.1]).

1.5. Обобщение теоремы 1.3 можно разбить на следующие, частично связанные, темы:

(А) рассматриваемые условия неизоморфности пространств должны быть безбазисными;

(В) требование сильного различия пространств должно быть ослаблено, возможность этого ослабления была изначально заложена в доказательстве теоремы 1.3.

Эта программа была частично реализована в предлагаемой статье:

(В) вместо компактных операторов рассматривается операторы, которые в работах Д. Фогта были названы ограниченными операторами (по терминологии Д. Фогта - bounded map);

(A) вместо классов $\left(d_{j}\right)$ рассматриваются классы пространств $\left(D_{j}\right)$, которые изначально имели безбазисное определение;

(C) на основе полученных в статье результатов (теорема 3.2 ) было доказано утверждение о неизоморфности весовых пространств непрерывных функций специального вида (теорема 4.4).

\section{2. Инвариантные классы.}

2.1. Пусть $X$ - пространство Фреше, $\left(\|\cdot\|_{p}\right)$ - монотонный набор норм, определяющий топологию в $X$. Говорят, что это пространство имеет тип $\left(D_{1}\right)=(D N)$ и пишут $X \in\left(D_{1}\right)=(D N)$, если

$$
\exists p_{0} \forall p \forall \tau \in(0,1) \exists p_{1}: \exists C>0 \forall x \in X\|x\|_{p} \leqslant C\|x\|_{p_{0}}^{\tau}\|x\|_{p_{1}}^{1-\tau} .
$$

Говорят, что это пространство имеет тип $\left(D_{2}\right)=(\bar{\Omega})$ и пишут $X \in\left(D_{2}\right)=(\bar{\Omega})$, если

$$
\forall q_{0} \forall \tau \in(0,1) \exists q \forall q_{1}: \exists C>0 \forall n\left\|x^{\prime}\right\|_{p}^{\prime} \leqslant C\left(\left\|x^{\prime}\right\|_{p_{0}}^{\prime}\right)^{1-\tau}\left(\left\|x^{\prime}\right\|_{p_{1}}^{\prime}\right)^{\tau} .
$$

Здесь $\left\|x^{\prime}\right\|_{s}:=\sup \left\{\left|x^{\prime}(x)\right|: x \in X,\|x\|_{s} \leqslant 1\right\}$.

Эти классы были определены в $[6,19,22]$.

В работах В. П. Захарюты в терминах инвариантных классов была построена частичная изоморфная классификация пространств аналитических и пространств Кёте специального вида степенных пространств Кёте первого и второго рода. Д. Фогт, М.-Й. Вагнер в терминах этих классов дали характеризацию подпространств и факторпространств в пространствах степенных рядов конечного и бесконечного типа. Основное отличие в подходах этих авторов заключается 
в том, что В. П. Захарюта определил классы пространств $\left(D_{j}\right)$ в терминах комплексных интерполяционных шкал. Непосредственно проверяется, что для пространства Кёте $K(A)$ утверждения $K(A) \in\left(d_{j}\right)$ и $K(A) \in\left(D_{j}\right)$ равносильны.

2.2. Пусть $X$ - пространство Фреше и $\left(U_{p}\right)$ - базис окрестностей нуля в $X$. Всегда можно считать, что $U_{p} \supset U_{p+1}$ для произвольного $p$.

Теорема 2.1. Принадлежность пространства $X$ классу $\left(D_{1}\right)$ равносильна каждому из следующих условий:

$$
\begin{gathered}
\exists p_{0} \forall p \forall d>0 \exists p_{1} \exists C>0: \forall x \in X \forall t>0\|x\|_{p} \leqslant t^{d}\|x\|_{p_{0}}+\frac{C}{t}\|x\|_{p_{1}}, \\
\exists p_{0} \forall p \forall d>0 \exists p_{1} \exists C>0: \forall t>0 U_{p}^{\circ} \subset t^{d} U_{p_{0}}^{\circ}+\frac{C}{t} U_{p_{1}}^{\circ},
\end{gathered}
$$

Теорема 2.2. Принадлежность пространства $X$ классу $\left(D_{2}\right)$ равносильна каждому из следующих условий:

$$
\begin{gathered}
\forall p_{0} \forall d>0 \exists p \forall p_{1} \exists C>0: \forall x^{\prime} \in X^{\prime} \forall t>0\left\|x^{\prime}\right\|_{p}^{\prime} \leqslant t^{d}\left\|x^{\prime}\right\|_{p_{0}}^{\prime}+\frac{C}{t}\left\|x^{\prime}\right\|_{p_{1}}^{\prime}, \\
\forall p_{0} \forall d>0 \exists p \forall p_{1} \exists C>0: \forall t>0 U_{p} \leqslant t^{d} U_{p_{0}}+\frac{C}{t} U_{p_{1}},
\end{gathered}
$$

Здесъ $\left(\|\cdot\|_{p}^{\prime}\right)$ - набор сопряжённых норм в $X^{\prime}$ :

$$
\left\|x^{\prime}\right\|_{p}^{\prime}:=\sup \left\{x^{\prime}(x):\|x\|_{p} \leqslant 1\right\}
$$

2.3. Пусть $X$ - пространство Фреше, $\left(X_{p}\right)$ - проективный спектр банаховых пространств, определяющих это пространство, $\|\cdot\|_{p}-$ норма в $X_{p}$. Покажем, что свойство пространства Фреше принадлежать инвариантным классам $\left(D_{j}\right)$ можно рассматривать как внутреннее интерполяционное свойство рассматриваемого пространства, которое формулируются в терминам метода вещественной интерполяции.

В теоремах 2.3-2.4 используются следующая интерпретация семейств, сопряжённых к интерполяционным семействам банаховых пространств [15]. Пусть даны банаховы пространства $E_{0}, E_{1}, \ldots$ и банахово пространство $E$, непрерывно и плотно вкладываются во все пространства $E_{j}$. Это замечание позволяет отождествлять пространство $E_{j}$ с пространством $E_{j}^{\sharp}$ :

$$
E_{j}^{\sharp}:=\left\{x^{\prime} \in E^{\prime}:|x|_{E}^{\sharp}:=\sup _{x \in E, x \neq 0} \frac{\left|x^{\prime}(x)\right|}{|x|_{E}}<+\infty\right\},
$$

наделённого нормой $|\cdot|_{E}^{\sharp}$. При таком отождествлении семейство $E_{0}^{\sharp}, E_{1}^{\sharp}, \ldots$ непрерывно вкладываются в одно и то же отделимое локально выпуклое пространство $E^{\prime}$. Тогда произвольный функционал $x^{\prime} \in E_{j}^{\sharp}$ однозначно определяется своим сужением на $E$. В частности, полярой $B^{\circ}$ подмножества $B$ в $E_{j}$ называют подмножество в $E_{j}^{\sharp}$ вида

$$
B^{\circ}:=\left\{y^{\prime} \in E^{\prime}: \forall y \in B\left|y^{\prime}(e)\right| \leqslant 1\right\} .
$$

В теоремах 2.3-2.5 предполагается, что даны такие банаховы пространства $X_{0}, X, X_{1}$ и векторное пространство $E$, что

(1) $X_{1} \subset X \subset X_{0}$ с плотными операторами вложения;

(2) $E$ всюду плотно во всех пространствах $X_{0}, X, X_{1}$;

(3) $\|\cdot\|_{0},\|\cdot\|,\|\cdot\|_{1}$ - нормы соответственно в $X_{0}, X, X_{1}$;

(4) $V_{0}, V, V_{1}$ - единичные шары соответственно в $X_{0}, X, X_{1}$;

(5) $U_{0}:=V_{0} \cap E, U:=V \cap E, U_{1}:=V_{1} \cap E$ - единичные шары соответственно в $\left(E,\|\cdot\|_{0}\right),(E,\|\cdot\|)$, $\left(E,\|\cdot\|_{1}\right)$.

Теорема 2.3. Следующие условия эквивалентны:

(1) $\exists \tau \in(0,1) \exists C>0 \forall x \in X_{1}\|x\| \leqslant C\|x\|_{0}^{1-\tau}\|x\|_{1}^{\tau}$; 
(2) $\exists \tau=\frac{1}{1+d} \in(0,1) \exists C>0 \forall t>0 \forall x \in X_{1}\|x\| \leqslant t^{d}\|x\|_{0}+\frac{C}{t}\|x\|_{1}$;

(3) $\exists \tau=\frac{1}{1+d} \in(0,1) \exists C>0 \forall t>0 U^{\circ} \subset t^{d} U_{0}^{\circ}+\frac{C}{t} U_{1}^{\circ}$;

(4) $\exists \tau=\frac{1}{1+d} \in(0,1) \forall p \in[1,+\infty) X \supset\left(X_{0}, X_{1}\right)_{\tau, p}$.

В условии (3) предполагается, что $B^{\circ}$ - поляра подмножества $B$.

Доказательство. Эквивалентность условий (1)-(3) (и равенство $\tau_{1}=\tau_{2}=\tau_{3}$ ) доказывается по схеме, разработанной в работах Д. Фогта [19]. Импликация (4) $\Rightarrow(1)$ следует из [9, теорема 1.3.3].

Предположим, что условие (3) выполняется при $\tau=\tau_{0}=\frac{1}{1+d_{0}}$. После подстановки $t=s^{d_{0}+1}$ условие (3) преобразуется к следующему виду:

$$
\forall s>0 U^{\circ} \subset s^{\tau_{0}} U_{0}^{\circ}+\frac{C}{s^{1-\tau_{0}}} U_{1}^{\circ} .
$$

Фиксируем произвольное $s>0$. Из (7) следует, что для произвольного $x^{\prime} \in U^{\circ}$ существует разложение

$$
x^{\prime}=s^{\tau_{0}} x_{0}^{\prime}+\frac{C}{s^{1-\tau_{0}}} x_{1}^{\prime},
$$

в котором $x_{j}^{\prime} \in U_{j}^{\circ}, j=0,1$. Из определения $K$-функционала следует, что

$$
K\left(x^{\prime}, s\right) \leqslant s^{\tau_{0}}\left\|x^{\prime}\right\|_{0}^{\prime}+C s \cdot s^{-\left(1-\tau_{0}\right)}\left\|x^{\prime}\right\|_{1}^{\prime} \leqslant C_{1} s^{\tau_{0}} .
$$

При $s \in(0,1]$ условие (8) можно уточнить. Для этого фиксируем произвольное $\varepsilon \in\left(0,\left(1-\tau_{0}\right) / 2\right)$. Существует число $C_{2}>0$ такое, что

$$
U^{\circ} \subset C_{2} U_{1}^{\circ} \subset C_{2} s^{2 \varepsilon-\left(1-\tau_{0}\right)} U_{1}^{\circ},
$$

$s \in(0,1]$. Из доказанного включения следует, что для произвольного $x^{\prime} \in U^{\circ}$ существует $x_{1}^{\prime} \in U_{1}^{\circ}$ такое, что

$$
K\left(x^{\prime}, s\right) \leqslant s \cdot C_{2} s^{2 \varepsilon-\left(1-\tau_{0}\right)}\left\|x^{\prime}\right\|_{1}^{\prime}=C_{2} s^{\tau_{0}+2 \varepsilon} .
$$

Но тогда для произвольных $q \in(1,+\infty)$ и $x^{\prime} \in U^{\circ}$ верна цепочка неравенств

$$
\left\|x^{\prime} \mid\left(X_{0}^{\sharp}, X_{1}^{\sharp}\right)_{\tau, q}\right\|^{q}=\int_{0}^{1}\left[s^{-\tau_{0}} K\left(x^{\prime}, s\right)\right]^{q} \frac{d s}{s}+\int_{1}^{+\infty}\left[s^{-\tau_{0}} K\left(x^{\prime}, s\right)\right]^{q} \frac{d s}{s} \leqslant \int_{0}^{1} s^{\varepsilon q} \frac{d s}{s}+\int_{1}^{+\infty} s^{-\varepsilon q} \frac{d s}{s}:=C_{4}<+\infty .
$$

Если же $q=\infty$, то

$$
\left\|x^{\prime} \mid\left(X_{0}^{\sharp}, X_{1}^{\sharp}\right)_{\tau, \infty}\right\|=\sup _{s>0} s^{-\tau_{0}} K\left(x^{\prime}, s\right) \leqslant \max \left\{\sup _{s \in(0,1]} s^{\varepsilon}, \sup _{s>1]} s^{-\varepsilon}\right\}:=C_{5}<+\infty .
$$

Из полученных неравенств следует, что

$$
\left\|x^{\prime}\left|\left(X_{0}, X_{1}\right)_{\tau, p}^{\sharp}\left\|\stackrel{(*)}{\leqslant} C_{6}\right\| x^{\prime}\right|\left(X_{0}^{\sharp}, X_{1}^{\sharp}\right)_{\tau, p}\right\| \leqslant\left\|x^{\prime}\right\|^{\prime}
$$

для произвольного $x^{\prime} \in X^{\prime}$. Неравенство $(*)$ есть следствие теоремы двойственности для вещественного метода интерполяции $[9,1.11 .2]$. Возможность применения этого утверждения следует из того, что по условию $X_{1}$ плотно вкладывается в $X_{0}$.

Из (10) следует, что $X^{\prime} \subset\left(X_{0}, X_{1}\right)_{\tau}^{\sharp}, p$. Искомое утверждение следует из леммы 2.1 .

Лемма 2.1. Пусть даны банаховы пространства $E_{0}, E_{1}$, промежуточные между $X_{0} u X_{1}$, причём $X_{1} \subset E_{j}$ с плотным образом оператора вложсения. Тогда следующие условия (а) $и$ (b) равносильны (операторы вложения в этих условиях имеют всюду плотный образ):

(a) $E_{1} \subset E_{0}$;

(b) $E_{0}^{\sharp} \subset E_{1}^{\sharp}$.

Теорема 2.4. Следующие условия эквивалентны:

(1) $\exists \tau \in(0,1) \exists C>0 \forall x \in X_{0}^{\prime}\left\|x^{\prime}\right\|^{\prime} \leqslant C\left(\left\|x^{\prime}\right\|^{\prime}\right)_{0}^{1-\tau}\left(\left\|x^{\prime}\right\|^{\prime}\right)_{1}^{\tau}$; 
(2) $\exists \tau=\frac{1}{1+d} \in(0,1) \exists C>0 \forall t>0 \forall x^{\prime} \in X_{0}^{\prime}\|x\|^{\prime} \leqslant t^{d}\left\|x^{\prime}\right\|_{1}^{\prime}+\frac{C}{t}\left\|x^{\prime}\right\|_{0}^{\prime}$;

(3) $\exists \tau=\frac{1}{1+d} \in(0,1) \exists C>0 \forall t>0 U \subset t^{d} U_{1}+\frac{C}{t} U_{0}$;

(4) $\exists \tau=\frac{1}{1+d} \in(0,1) \forall p \in[1,+\infty) X \subset\left(X_{1}, X_{0}\right)_{\tau, p}$.

Если $\tau_{j}$ - наилучшие значение числе $\tau$ в условии $j$, то $\tau_{1}=\tau_{2}=\tau_{3}=\tau_{4}$.

Доказательство. Как и в доказательстве теоремы 2.4, в доказательстве нуждается только эквивалентность условий (3) и (4).

Докажем импликацию $(4) \Rightarrow(1)$. Для этого перейдём в условии (4) к сопряжённым пространствам:

$$
\left(X_{1}^{\sharp}, X_{0}^{\sharp}\right)_{\tau, q}=\left(X_{1}, X_{0}\right)_{\tau, q}^{\sharp} \subset X^{\sharp}, \quad p^{-1}+q^{-1}=1 .
$$

Тогда условие (1) (и равносильное ему условие (3)) есть следствие [9, теорема 1.3.3].

Доказательство импликации $(3) \Rightarrow(4)$ дословно повторяет доказательство импликации $(3) \Rightarrow(4)$ из теоремы 2.3.

В теоремах 2.3 и 2.4 условия (4) формулируются в терминах пространств $\left(E_{0}, E_{1}\right)_{\tau, p}$ с конечным $p$. Ограничение обусловлено использование теорем действенности и плотности для метода вещественной интерполяции.

Для произвольной интерполяционной пары банаховых пространств $\left[E_{0}, E_{1}\right]$ обозначим через $\left(E_{0}, E_{1}\right)_{\tau, \infty}^{\circ}$ замыкание $E_{0} \cap E_{1}$ в топологии пространства $\left(E_{0}, E_{1}\right)_{\tau, \infty}$.

Теорема 2.5. Справедливы импликации $(1) \Rightarrow(2) \Rightarrow(3)$, где

(1) $\exists \tau=\frac{1}{1+d} \in(0,1) X \supset\left(X_{0}, X_{1}\right)_{\tau, \infty}$.

(2) $\exists \tau=\frac{1}{1+d} \in(0,1) \exists C>0 \forall t>0 U^{\circ} \subset t^{d} U_{0}^{\circ}+\frac{C}{t} U_{1}^{\circ}$;

(3) $\exists \tau=\frac{1}{1+d} \in(0,1) X \supset\left(X_{0}, X_{1}\right)_{\tau, \infty}^{\circ}$.

и импликации $(4) \Rightarrow(5) \Rightarrow(6)$, где

(4) $\exists \tau=\frac{1}{1+d} \in(0,1) X \subset\left(X_{1}, X_{0}\right)_{\tau, \infty}^{\circ}$,

(5) $\exists \tau=\frac{1}{1+d} \in(0,1) \exists C>0 \forall t>0 U \subset t^{d} U_{1}+\frac{C}{t} U_{0}$;

(6) $\exists \tau=\frac{1}{1+d} \in(0,1) X \subset\left(X_{1}, X_{0}\right)_{\tau, \infty}$.

\section{3. Ограниченные операторы.}

3.1. Возможность обобщения теоремы 1.3 изначально содержалась в доказательстве этого утверждения. Заметим, что условие монтелевости пространства $K(B)$ применялось только на последнем этапе доказательства, на котором из ограниченности подмножества в пространстве $K(B)$ выводилась относительно компактность этого множества. Это замечание приводит к следующему определению:

Определение 3.1 (см. [20], [16, с. 375]). Линейный оператор $T: X \rightarrow Y$ называют ограниченным (по терминологии авторов - bounded map), если существует окрестность нуля $U$ в $X$ такая, что множество $T U$ ограничено в $Y$.

Тогда верно следующие утверждение.

Теорема 3.1 (см. [23]). Пусть выполняются следующие условия:

(1) $X \cong K(A), Y \cong K(B)$;

(2) $K(A) \in\left(d_{2}\right), K(B) \in\left(d_{1}\right)$.

Тогда любой непрерывный линейный оператор $T: X \rightarrow Y$ ограничен. Если, кроме того, эти пространства не нормируемые, то они не изоморфны. 
Следует отметить, что свойство линейного оператора $T: X \rightarrow Y$ быть ограниченным нетривиально только для ненормируемых пространств $X, Y$.

Теорема 3.2 (см. [10]). Пусть $X, Y$ - пространства Фреше и $X \in\left(D_{2}\right), X \in\left(D_{1}\right)$. Тогда любой непрерывный линейный оператор $T: X \rightarrow Y$ ограничен. Если, кроме того, эти пространства не нормируемые, то они не изоморфны.

Доказательство. Пусть выполняются все условия теоремы. Тогда из определения классов $\left(D_{j}\right)$ и теорем 2.3 и 2.4 следует, что:

$$
\begin{gathered}
\exists p_{0} \forall q_{0} \exists q=q\left(q_{0}\right)>q_{0} \forall p>p_{0} \exists p_{1}=p_{1}(p)>p \forall q_{1}>q \\
\|x\|_{2, p} \leqslant C_{1}\left\|y\left|\left(Y_{1, p_{1}}, Y_{1, p_{0}}\right)_{1 / 3,2}\left\|=C_{1}\right\| y\right|\left(Y_{1, p_{0}}, Y_{1, p_{1}}\right)_{2 / 3,2}\right\|, \\
\left\|x \mid\left(X_{1, p_{0}}, X_{1, p_{1}}\right)_{2 / 3,2}\right\| \leqslant C_{2}\|x\|_{0, p}, \\
\left\|T x\left|Y\left\|_{p_{j}} \leqslant\right\| x\right| X\right\|_{q_{j}}
\end{gathered}
$$

для произвольных $x \in X, y \in Y$. Фиксируем индексы $p_{0}, p, q_{0}$ так, чтобы выполнялись условия (11), (12) При этом всегда можно считать, что $p_{0}<q_{0}, p_{1}<q_{1}$. Таким образом, индекс $q$ зависит только от $q_{0}$, который зависит только от $p_{0}$.

Но тогда для произвольного $x \in X_{0}$ выполняется неравенство

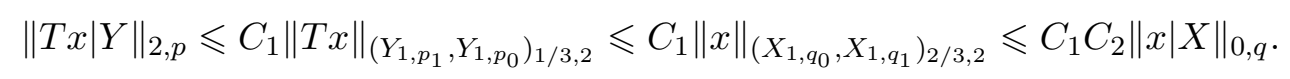

Из сделанных предположений относительно индексов норм $p_{0}, p, p_{1}$ и $q_{0}, q, q_{1}$ и предыдущего неравенства следует ограниченность оператора $T$. Теорема доказана.

\section{4. Весовые пространства непрерывных функций.}

4.1. Пусть $D \subset \mathbb{R}^{n}$ - область (т.е. открытое и связное множество). Функцию $F: D \rightarrow \mathbb{R}$ будем называть весовой (точнее вещественной весовой функцией, другие варианты определения весовой функции в пространствах непрерывных функций см. в $[1,13,14]$, если она непрерывна. Из определения весовой функции следует, что

$$
\forall x \in D \exists R=R_{F}(x)>0: \forall y \in D,\|x-y\|_{\mathbb{R}^{n}}<R|F(x)-F(y)|<1
$$

для произвольной функции $F \in \mathcal{C}$. Всюду $L$ с различными добавочными индексами - числа, не зависящие от функции $f$.

Если $F$ - весовая функция, то через $C(D, F)$ обозначим множество всех непрерывных функций $f: D \rightarrow \mathbb{R}$, для которых конечна норма

$$
\|f\|_{F}:=\sup _{x \in D}|f(x)| e^{-F(x)} .
$$

Лемма 4.1. При сделанных предположениях $C(D, F)$ - банахово пространство относительно норми $\|\cdot\|_{F}$.

Через $C_{0}(D, F)$ обозначим множество всех $f \in C(D, F)$, для которых $|f(x)|=O\left(e^{F(x)}\right)$ при $x \rightarrow \partial D$. Другими словами, $f \in C_{0}(D, F)$ тогда и только тогда, когда

$$
\forall \varepsilon>0 \exists K \Subset D:|f(x)| \leqslant \varepsilon e^{F(x)} \quad \forall x \in D \backslash K .
$$

По построению, $C_{0}(D, F)$ есть замкнутое подпространство в $C(D, F)$. Необходимость рассмотрения пространств $C_{0}(D, F)$ обусловлена следующими утверждениями.

Лемма 4.2. Множество непрерывных функиий $f: D \rightarrow \mathbb{R}$ с компактным носителем всюду плотно в $C_{0}(D, F)$.

Как обычно, носителем $\operatorname{supp}(f)$ непрерывной функции $f$ называют замыкание подмножества в $D$, на котором функция не обращается в ноль.

Лемма 4.3. Следуюшие условия равносильны для любых весовых функиий $G_{0} \in \mathcal{C}_{D}, G_{1} \in \mathcal{C}_{D}$ : (1) $\exists R>0: \forall x \in D G_{1}(X) \leqslant G_{0}(x)+R$; 
(2) $C\left(D, G_{1}\right) \subset C\left(D, G_{0}\right)$;

(3) $C_{0}\left(D, G_{1}\right) \subset C_{0}\left(D, G_{0}\right)$.

Лемма 4.4. Пусть $F_{0}, F_{1}$ - такие весовъе функции в области $D$, что $F_{1}(x)<F_{0}(x)$ в $D$ и $F_{1}(x)-F_{0}(x) \rightarrow-\infty$ при $x \rightarrow \partial D$. Тогда $C_{0}\left(D, F_{1}\right)$ есть всюду плотное векторное подпространство в $C_{0}\left(D, F_{0}\right)$.

4.2. Пусть $\mathcal{F}=\left(F_{p}\right)$ - семейство вещественных весовых функций в области $D$. Будем называть это семейство весовым (точнее, вещественным весовым семейством), если

(1) $F_{p+1}(x) \leqslant F_{p}(x)$ для всех $x \in D$;

(2) $F_{p}(x)-F_{p+1}(x) \rightarrow+\infty, x \rightarrow \partial D$

для произвольного $p$. Множество всех вещественных весовых семейств обозначим через $\left(\mathcal{C}_{D}\right)$.

Если $\mathcal{F}=\left(F_{p}\right) \in\left(\mathcal{C}_{D}\right)$, то $C(D, \mathcal{F})$ - множество всех непрерывных функций $f: D \rightarrow \mathbb{R}$, для которых конечны все нормы $\|\cdot\|_{p},\|\cdot\|_{p}:=\|\cdot\|_{F_{p}}$.

Лемма 4.5. При сделанных предположениях $C(D, \mathcal{F})$-пространство Фреше, топология в котором определяется набором норм $\left(\|\cdot\|_{p}\right)$. Кроме того, пополнение $C(D, \mathcal{F})$ по норме $\|\cdot\|_{p}$ совпадает с $C_{0}\left(D, F_{p}\right)$.

4.3. Изоморфная классификация пространств аналитических функций, построенная В. П. Захарютой (см. $[3,4,6])$, существенно опирается на изучение интерполяционных свойств пространств этого типа. В свою очередь, интерполяционные свойства пространств аналитических функций есть следствие теоремы о шкале пространств аналитических функций и аналитических функционалов (см. [5]).

Если опустить некоторые технические детали, то теорему В. П. Захарюты о шкалах можно сформулировать следующим образом: пусть даны область $\Omega \subset \mathbb{C}^{n}$ и компакт $K \Subset \Omega$. При выполнении ряда дополнительных условий найдутся такие плюрисубгармоническая функция $u: D \rightarrow \mathbb{R}$ и сепарабельные гильбертовы пространства $H_{0}, H_{1}$, что $A(\bar{D}) \subset H_{1} \subset A(D) \subset A(K) \subset H_{0} \subset$ $A C(K)$. Тогда

$$
A\left(K_{\alpha}\right) \subset\left(H_{0}\right)^{1-\alpha}\left(H_{1}\right)^{\alpha} \subset A\left(D_{\alpha}\right)
$$

для произвольного $\alpha \in(0,1)$. В (14) используются следующие обозначения:

(1) $D_{\alpha}:=\{z \in D: u(z)<\alpha\}$

(2) $A(\Omega)$ - пространство функций, аналитических в области $\Omega$, наделённое топологией равномерной сходимости на компактных подмножествах в $\Omega$;

(3) $K_{\alpha}:=\{z \in D: u(z) \leqslant \alpha\}$;

(4) если $K \Subset \Omega$, то $A(K)$ состоит из функций, аналитических на множестве $K$;

(5) $\left[\left(H_{0}\right)^{1-\alpha}\left(H_{1}\right)^{\alpha}\right]$ - комплексная шкала, построенная по пространствам $H_{0}$ и $H_{1}$.

В статье доказывается аналог теоремы В. П. Захарюты о шкале, в котором рассматриваются семейства весовых банаховых пространств непрерывных функций. Основное отличие заключается в том, что вместо метода комплексных шкал применяется метод вещественной интерполяции.

Теорема 4.1. Пусть $F_{0}, F_{1}$ - весовые функиии в области $D$ такие, что $F_{1}(x)<F_{0}(x)$ для произвольного $x \in D$. Положим $F_{\tau}:=(1-\tau) F_{0}+\tau F_{1}$. Тогда

$$
C\left(D, F_{\tau+\varepsilon}\right) \subset\left(C\left(D, F_{0}\right), C\left(D, F_{1}\right)\right)_{\tau, \infty} \subset C\left(D, F_{\tau}\right)
$$

для любого $\tau \in(0,1) u \varepsilon \in(0,1-\tau)$.

Доказательство теоремы 4.1 разбивается на две части-более простую (лемма 4.6) и более сложную (лемма 4.7). Возможность доказательства теоремы 4.1 связана с тем, что пространства $C\left(D, F_{0}\right)$ и $C\left(D, F_{1}\right)$ имеют много общих бесконечномерных пространств.

Лемма 4.6. Пусть $F_{0}, F_{1}$ - весовые функиии в области $D$ mакие, что $F_{1}(x)<F_{0}(x)$ для произвольного $x \in D$. Тогда

$$
\left(C\left(D, F_{0}\right), C\left(D, F_{1}\right)\right)_{\tau, \infty} \subset C\left(D, F_{\tau}\right)
$$

для любого $\tau \in(0,1)$. 
Доказательство. Для доказательства непрерывности вложения (16) фиксируем произвольную ненулевую функцию $f$, принадлежащую левой части условия (16). Из определения $K$-функционала следует, что для произвольного положительного $t$ существование разложения $f=f_{0}+f_{1}$ такое, что $f_{j} \in C\left(D, F_{j}\right), j=0,1$ и $K(f, t) \leqslant\left\|f_{0}\right\|_{F_{0}}+t\left\|f_{1}\right\|_{F_{1}} \leqslant 2 K(f, t)$. Но тогда для любого $x \in D$ такого, что $f(x) \neq 0$, справедлива следующая цепочка неравенств:

$$
\begin{aligned}
2 K(f, t) \geqslant & \left\|f_{0}\right\|_{F_{0}}+t\left\|f_{1}\right\|_{F_{1}} \geqslant\left|f_{0}(x)\right| e^{-F_{0}(x)}+t\left|f_{1}(x)\right| e^{-F_{1}(x)}= \\
& =\left(\left|f_{0}(x)\right|+\left|f_{1}(x)\right|\right)\left(\frac{\left|f_{0}(x)\right|}{\left|f_{0}(x)\right|+\left|f_{1}(x)\right|} e^{-F_{0}(x)}+t \frac{\left|f_{1}(x)\right|}{\left|f_{0}(x)\right|+\left|f_{1}(x)\right|} e^{-F_{1}(x)}\right) \geqslant \\
& \geqslant|f(x)| \inf _{\tau \in[0,1]}\left((1-\tau) e^{-F_{0}(x)}+\tau t e^{-F_{1}(x)}\right)=|f(x)| \min \left\{e^{-F_{0}(x)}, t e^{-F_{1}(x)}\right\} .
\end{aligned}
$$

Таким образом, доказано, что $2 K(f, t) \geqslant|f(x)| \min \left\{e^{-F_{0}(x)}, t e^{-F_{1}(x)}\right\}$ для произвольной функции $f \in\left(C\left(D, F_{0}\right), C\left(D, F_{1}\right)\right)_{\tau, \infty}$. Но тогда

$$
\left\|f\left|\left(C\left(D, F_{0}\right), C\left(D, F_{1}\right)\right)_{\tau, \infty} \| \geqslant \frac{1}{2} \sup _{t>0}\left[t^{-\tau}|f(x)| \min \left\{e^{-F_{0}(x)}, t e^{-F_{1}(x)}\right\}\right]=\frac{1}{2}\right| f(x) \mid e^{-F_{\tau}(x)} .\right.
$$

Если в полученном неравенстве перейти к точной по всем $x \in D$, то получается неравенство

$$
\|f\|_{F_{\tau}}=\sup _{x \in D}|f(x)| e^{-F_{\tau}(x)} \leqslant 2\left\|f \mid\left(C\left(D, F_{0}\right), C\left(D, F_{1}\right)\right)_{\tau, \infty}\right\|,
$$

из которого следует, что $f \in C\left(D, F_{\tau}\right)$. Но тогда $\left(C\left(D, F_{0}\right), C\left(D, F_{1}\right)\right)_{\tau, \infty}$ непрерывно вкладывается в $C\left(D, F_{\tau}\right)$. Лемма доказана.

Лемма 4.7. Пусть $F_{0}, F_{1}$-весовые функиии в области $D$-удовлетворяют условиям теоремы 4.1. Тогда

$$
C\left(D, F_{\tau+\varepsilon}\right) \subset\left(C\left(D, F_{0}\right), C\left(D, F_{1}\right)\right)_{\tau, \infty}
$$

для любого $\tau \in(0,1) u \varepsilon \in(0,1-\tau)$.

Доказательство. Для произвольной ненулевой функции $f \in C\left(D, F_{1}\right)$ положим

$$
\Phi(f)=\Phi\left(f ; F_{0}, F_{1}\right):=\inf _{x \in D, f(x) \neq 0} \max _{j=0,1} \frac{\|f\|_{F_{j}}}{|f(x)| e^{-F_{j}(x)}} .
$$

Из определения функционала $\Phi(\cdot)$ и метода вещественной интерполяции следует, что

$$
\forall f \in C\left(D, F_{1}\right) \quad\left\|f \mid\left(C\left(D, F_{0}\right), C\left(D, F_{1}\right)\right)_{\tau, \infty}\right\| \leqslant 2 \Phi(f)\|f\|_{\tau},
$$

где $\operatorname{supp}(f)$ - носитель функции $f$ (т.е. замыкание в $\mathbb{R}^{n}$ множества всех $x \in D$, где $\left.f(x) \neq 0\right)$.

Фиксируем произвольные $\tau \in(0,1)$ и $\varepsilon \in(0,1-\tau)$. Предположим, что для произвольной ненулевой функции $f \in C\left(D, F_{\tau+\varepsilon}\right)$ найдено такое разложение $f=\sum_{s=0}^{+\infty} f_{s}$, что

(a) $f_{s} \in C\left(D, F_{1}\right)$;

(b) числовая последовательность $\left(\Phi\left(f_{s}\right)\right)$ ограничена;

(c) существует число $L_{1}$ такое, что

$$
\sum_{s=0}^{+\infty} \frac{\left\|f_{s}\right\|_{\tau}}{\left\|f_{s}\right\|_{\tau+\varepsilon}} \leqslant L_{1}
$$

(d) $\left\|f_{s}\right\|_{\tau+\varepsilon} \leqslant\|f\|_{\tau+\varepsilon}$.

Тогда

$$
\begin{aligned}
\left\|f \mid\left(C\left(D, F_{0}\right), C\left(D, F_{1}\right)\right)_{\tau, \infty}\right\| & \leqslant \sum_{s=0}^{+\infty}\left\|f_{s} \mid\left(C\left(D, F_{0}\right), C\left(D, F_{1}\right)\right)_{\tau, \infty}\right\| \leqslant \\
& \leqslant 2 \sum_{s=0}^{+\infty} \Phi\left(f_{s}\right)\left\|f_{s}\right\|_{\tau} \leqslant L_{2} \sum_{s=0}^{+\infty}\left\|f_{s}\right\|_{\tau+\varepsilon} \frac{\left\|f_{s}\right\|_{\tau}}{\left\|f_{s}\right\|_{\tau+\varepsilon}} \leqslant L_{3}\|f\|_{\tau+\varepsilon}
\end{aligned}
$$


Докажем, что для произвольной ненулевой функции $f \in C\left(D, F_{\tau+\varepsilon}\right)$ найдётся разложение, удовлетворяющее условиям (a)-(d). Пусть $\left(\lambda_{s}\right)_{s \geqslant 0}$ - такая числовая последовательность, что $\lambda_{0}=1$, $\lambda_{s} \downarrow 0$ и $\lambda_{s+1} \asymp \lambda_{s}$ при $s \uparrow+\infty, \sum_{s=0}^{+\infty} \lambda_{s}^{\varepsilon}<+\infty$ для произвольного $\varepsilon>0$.

Положим $K_{s}:=\left\{x \in D: \ln \left(\lambda_{s+1}\right)<F_{1}(x)-F_{0}(x) \leqslant \ln \left(\lambda_{s}\right)\right\}, s \geqslant 0$. По построению множества $K_{s}$ попарно не пересекаются и $\bigcup_{s \geqslant 0} K_{s}=D$. Из свойств последовательности $\left(\lambda_{s}\right)$ следует существование такого числа $L_{4}>0$, что

$$
\ln \frac{\lambda_{s}}{L_{4}} \leqslant F_{1}(x)-F_{0}(x) \leqslant \ln \left(L_{4} \lambda_{s}\right)
$$

для произвольных $s \geqslant 0$ и $x \in \Omega_{s}:=\bigcup_{x \in K_{s}} B(x, R(x))$, где $R(x):=\min \left\{R_{F_{0}}(x), R_{F_{1}}(x)\right\}>0$, $B(x, r)$ - открытый шар в $\mathbb{R}^{n}$ с центром в точке $x$ радиуса $r$, отображение $x \mapsto R_{F}(x)$ определяется условием (13) по весовой функции $F$. Существуют такие последовательность $\left(x_{k}\right)$ точек из $D$ и семейство множеств $\left(N_{s}\right)_{s \geqslant 0}$, что

(е) множества $N_{s}$ попарно не пересекаются и $\bigcup_{s \geqslant 0} N_{s}=\mathbb{N}$;

(f) семейство $\left\{B\left(x_{k}, R\left(x_{k}\right)\right)\right\}$ образует локально конечное открытое покрытие области $D$ (т.е. каждая точка $x \in D$ содержится в конечном множестве шаров $\left.B\left(x_{k}, R\left(x_{k}\right)\right)\right)$;

(g) $x_{k} \in K_{s}, k \in N_{s}$.

По семейству шаров строится такое семейство гладких функций $\left(\psi_{k}\right)$, что

(h) $0 \leqslant \psi_{k}(x) \leqslant 1$ в $D$;

(i) $\operatorname{supp}\left(\psi_{k}(x)\right) \subset B\left(x_{k}, R\left(x_{k}\right)\right)$;

(j) $\sum_{k} \psi_{k}(x) \equiv 1$ в $D$.

Наконец, положим $\Psi_{s}:=\sum_{k \in N_{s}} \psi_{k}$. По построению $\Psi_{s}(x) \geqslant 0, \operatorname{supp}\left(\Psi_{s}\right) \subset \Omega_{s}, \sum_{s \geqslant 0} \Psi_{s} \equiv 1$ в $D$. Из условий $(\mathrm{h})-(\mathrm{j})$ следует, что разложение $f=\sum_{s \geqslant 0} f \Psi_{s}$ удовлетворяет условиям (a)-(d).

Таким образом, доказано существование такого числа $L>0$, зависящего только от $\tau \in(0,1)$ и $\varepsilon \in(0,1-\tau)$, что $\left\|f \mid\left(C\left(D, F_{0}\right), C\left(D, F_{1}\right)\right)_{\tau, \infty}\right\| \leqslant L\|f\|_{\tau+\varepsilon}$ для произвольной функции $f \in$ $C\left(D, F_{\tau+\varepsilon}\right)$. Но тогда $C\left(D, F_{\tau+\varepsilon}\right)$ непрерывно вкладывается в $\left(C\left(D, F_{0}\right), C\left(D, F_{1}\right)\right)_{\tau, \infty}$. Лемма доказана.

Теорема 4.2. Пусть $F_{0}, F_{1}$ - такие весовые функиии в области $D$, что $F_{1}(x)<F_{0}(x)$ для произвольного $x \in D$ и $F_{0}(x)-F_{1}(x) \rightarrow+\infty$ при $x \rightarrow \partial D$. Тогда

$$
C_{0}\left(D, F_{\tau+\varepsilon}\right) \subset\left(C_{0}\left(D, F_{0}\right), C_{0}\left(D, F_{1}\right)\right)_{\tau, \infty}^{\circ} \subset\left(C_{0}\left(D, F_{0}\right), C_{0}\left(D, F_{1}\right)\right)_{\tau, \infty} \subset C_{0}\left(D, F_{\tau-\varepsilon}\right)
$$

для любого $\tau \in(0,1)$ и $0<\varepsilon<\min (\tau, 1-\tau)$.

4.4. Условие принадлежности пространства $C(D, \mathcal{F})$ классам $\left(D_{1}\right)$ и $\left(D_{2}\right)$ можно сформулировать непосредственно в терминах весового семейства $\mathcal{F}$.

Теорема 4.3. Пусть $\mathcal{F}=\left(F_{p}\right)$ - весовое семейство функиий. Тогда следующие условия (1)(2), (3)-(4) попарно эквивалентны:

(1) $C(D, \mathcal{F}) \in\left(D_{1}\right)$;

(2) $\exists p_{0} \forall p \forall \tau \in(0,1) \exists p_{1} \forall y \in D F_{p}(y) \geqslant-C+(1-\tau) F_{p_{0}}(y)+\tau F_{p_{1}}(y)$;

(3) $C(D, \mathcal{F}) \in\left(D_{2}\right)$

(4) $\forall p_{0} \forall \tau \in(0,1) \exists p \forall p_{1} \forall y \in D F_{p}(y) \leqslant C+\tau F_{p_{0}}(y)+(1-\tau) F_{p_{1}}(y)$.

Доказательство. Доказательство эквивалентности условий (1)-(2) есть следствие локализуемости свойств непрерывных функций и не нуждается в применении теоремы 4.2.

Пусть $C(D, \mathcal{F}) \in\left(D_{1}\right)$, т.е.

$$
\exists p_{0} \forall p \exists \tau \in(0,1) \exists p_{1} C_{0}\left(D, F_{p}\right) \supset\left(C_{0}\left(D, F_{p_{0}}\right), C_{0}\left(D, F_{p_{1}}\right)\right)_{\tau, \infty}^{\circ}
$$


Условие (18) есть следствие определения класса $\left(D_{2}\right)$, теоремы 2.5. Из теоремы 4.2 и (18) следует, что

$$
C_{0}\left(D, F_{p}\right) \supset\left(C_{0}\left(D, F_{p_{0}}\right), C_{0}\left(D, F_{p_{1}}\right)\right)_{\tau, \infty}^{\circ} \supset C_{0}\left(D,\left(1-\tau^{\prime}\right) F_{p_{0}}+\tau^{\prime} F_{p_{1}}\right), \quad \tau^{\prime}=\tau+\varepsilon
$$

для произвольного $\varepsilon \in(0,1-\tau)$. Но тогда из леммы 4.3 следует условие (6). Обратно, из условия (2) и теоремы 4.2 следует, что

$$
\exists p_{0} \forall p \exists p_{1} \exists \tau \in(0,1) C_{0}\left(D, F_{p}\right) \supset C_{0}\left(D,(1-\tau) F_{p_{0}}+\tau F_{p_{1}}\right) \supset\left(C_{0}\left(D, F_{p_{0}}\right), C_{0}\left(D, F_{p_{1}}\right)\right)_{\tau, \infty} .
$$

Но тогда условие (1) есть следствие теоремы 2.5 .

\section{5. Пусть}

(1) $a: D \rightarrow \mathbb{R}$ - такая непрерывная функция, что $a(x) \rightarrow+\infty, x \rightarrow \partial D$;

(2) $-\infty \leqslant \delta<+\infty$,

(3) $\left(\delta_{p}\right), \delta_{p+1}<\delta_{p}$ для произвольного $p$.

Семейство функций $\mathcal{F}=\left(\delta_{p} a\right)$ будет весовым. Положим $C_{\delta}(D, a)=C(D, \mathcal{F})$. Пространство $C_{\delta}(D, a)$ не зависит от выбора последовательности $\left(\delta_{p}\right)$.

Теорема 4.4. При сделанных предположениях $C_{\infty}(D, a) \in\left(D_{1}\right), C_{0}(D, a) \in\left(D_{2}\right)$.

Теорема 4.5. При сделанных предположения справедливы следующие утверждения:

(1) пространства $C_{\delta}(D, a)$ и $C_{0}(D, a)$ изоморфны для произвольного конечного $\delta$;

(2) произвольный линейный непрерывный оператор $T: C_{0}(D, a) \rightarrow C_{-\infty}(D, a)$ ограничен;

(3) пространства $C_{-\infty}(D, a)$ и $C_{0}(D, a)$ всегда не изоморфнъ.

\section{СПИСОК ЛИТЕРАТУРЫ}

1. Абанин A. В. Весовые пространства непрерывных и голоморфных функций// Тр. Междунар. конф. «Математический анализ и математическое моделирование». - Владикавказ: ЮМИ ВНЦ РАН, 2010. - C. $15-20$.

2. Драгилев М. М. О правильных базисах в ядерных пространствах// Мат. сб. - 1965. - 68, № 3. C. $153-173$.

3. Захарюта В. П. Об изиморфизме декартовых произведений линейных топологических пространств// Функц. анал. прилож. - 1970. - 4, № 2. - С. 87-89.

4. Захарюта В. П. Некоторые линейные топологические инварианты и изоморфизм тензорных произведений центров шкал// Изв. СКНЦ ВШ. Сер. естеств. наук. - 1974. - 4. - С. 62-64.

5. Захарюта В. П. Экстремальные плюрисубгармонические функции, гильбертовы шкалы и изоморфизм пространств аналитических функций многих переменных, // Теор. функций, функц. анал. прилож. (Харьков). - 1974. - 19. - С. 133-157.

6. Захарюта В. П. Изоморфизмы пространств аналитических функций// Докл. АН СССР. - 1980. 255, № 1. - C. 11-14.

7. Колмогоров А. Н. О линейной размерности топологических векторных пространств// Докл. АН CCCP. - 1958. - 120, № 2. - C. 239-241.

8. Митягин Б. С. Эквивалентность базисов в гильбертовых шкалах// Stud. Math. - 1971. - 37. C. $111-137$.

9. Трибель X. Теория интерполяции, Функциональные пространства. Дифференциальные операторы. М.: Мир, 1980.

10. Шубарин М. А. Операторные идеалы, определяемые инвариантными классами пространств Фреше// Мат. форум (Итоги науки. Юг России). - 2013. - 8. - С. 100-112.

11. Bessaga C., Pelczynski A., Rolewciz S. On diametral approximative dimension und linear homogeneity of F-spaces// Bull. Acad. Pol. Sci. - 1961. - 9. - P. 677-683.

12. Bessaga C., Pelczynski A., Rolewicz S. Approximative dimension of liner topological spaces and some of its applications// Stud. Math., Ser. Spec. - 1963. - 1. - P. 26-29.

13. Bierstedt K. D., Bonet J. Weighted ( $L F)$-spaces of continuous functions// Math. Nachr. - 1994. - 165. - P. 25-48.

14. Bierstedt K. D., Meise R., Summers V. H. A projective descriptions of weighted inductive limits// Trans. Am. Math. Soc.. - 272, № 1. - P. 107-160. 
15. Cwikel M. Lecture notes on duality and interpolation spaces/ arXiv:0803.3558v2 [math.FA].

16. Meise R., Vogt D. Introduction to Functional Analysis. - Oxford: Clarendon Press, 1997.

17. Pelczynski A. On the approximation of S-spaces by finite dimension space// Bull. Acad. Pol. Sci. - 1957. - 5, № 9. - P. 879-881.

18. Rolewicz S. On spaces of holomorphic functions// Stud. Math. — 1962. - 21, № 2. - P. 135-160.

19. Vogt D. Charakterisierung der Unterräume vons// Math. Z. - 1977. - 155. — P. 109-117.

20. Vogt D. Frecheträume, zwishen denen jede stetige linear Abbildung beschränkt ist// J. Reine Angew. Math. - 1983. - 345. - P. 182-200.

21. Vogt D., Wagner M. J. Charakterisierung der Unterräume und Quotientenräume der nuclearen stabilen Potenzreihenräumen von unendlichem Typ// Stud. Math. — 1981. — 70. — P. 63-80.

22. Wagner M.-J. Quotientenräume von stabilen Potenzreihenrümen endlichen Typs// Manuscr. Math. — 1980. - 31. - P. 97-109.

23. Zachariuta V. P. On isomorphism of Cartesian products of local convex spaces// Stud. Math. — 1973. 46. - P. 201-221.

24. Zahariuta $V$. Linear topologic invariants and their applications to isomorphic classification of generalized power spaces// Tr. J. Math. — 1996. - 20. - P. 237-289.

Шубарин Михаил Александрович

Южный федеральный университет, Ростов-на-Дону

E-mail: mas102@mail.ru 